\title{
What Are the Implications of REACH?
}
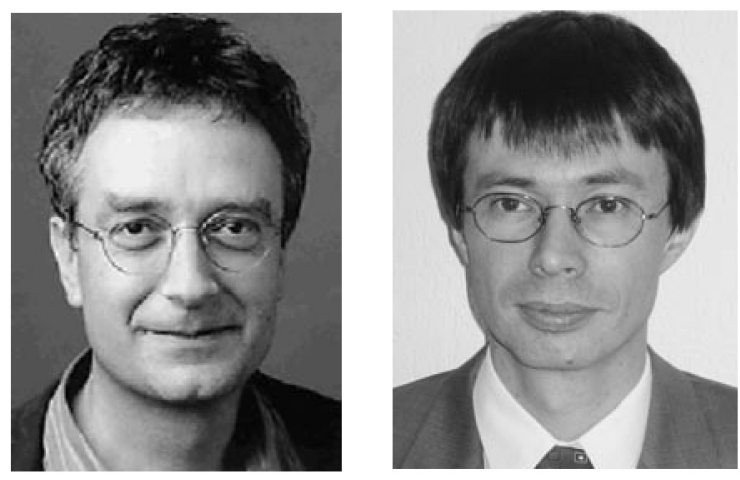

Chemical products are essential for the quality of life today and at the same time the raison d'être of the chemical industry. Risks of chemical products are, on the one hand, characterized by large, sometimes huge production volumes and, on the other hand, by the chemicals' enormous diversity in terms of molecular design. To establish a more comprehensive legal framework for dealing with the risks of chemicals, the new chemicals legislation of the EU, REACH - Registration, Evaluation and Authorization of CHemicals, is being developed. REACH will substantially change the practice of chemical risk assessment in the EU and in countries delivering chemicals to the EU; it will also create a new demand for efficient methods in all fields of chemical risk assessment and management. For Switzerland, a country with a strong chemical industry located in the heart of Europe, the consequences of REACH are without doubt of great importance.

$\mathrm{REACH}$ will probably enter into force in 2007 , but the general shape of the new legislation is already visible. Therefore, we asked, for this issue of CHIMIA, several representatives from governments, industry and academia to present their current view on REACH. The first four contributions represent perspectives of authority representatives from the EU, Germany and of course from Switzerland. These contributions summarize the current status of the REACH legislation, point out open questions and discuss implications for Switzerland. They are followed by four industry viewpoints presented by the Swiss chemical industry and a Swiss insurance company. The last part contains three scientific contributions; two of them focus on methods for estimating various chemical properties needed in chemical risk assessment and the last one evaluates the treatment of uncertainty and missing knowledge under REACH.

Of course, the contributions are all based on present knowledge and cannot provide a final evaluation of REACH and its implications. We are only at the beginning of a long process of learning what the consequences of REACH will be. However, the contributions presented here point out issues and aspects that will be important in the discussions about $\mathrm{REACH}$ in the next years. We hope that this special issue of CHIMIA will help to understand the different perspectives of the different actors involved. These actors represent a broad spectrum of perspectives, ranging from the political realm where different and often conflicting values and legal requirements have to be balanced to the domain of business where decisions about products and investments have to be made in a competitive environment, and finally to the domain of research where the risks of chemicals to safety, health and the environment are investigated in a more fundamental way. The different contributions to this issue shed light on needs for improvements of the new legislation, needs for improving the management of chemicals by companies, and on research needs leading to improved scientific methods for chemical risk assessment.

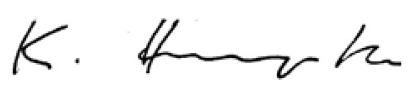

Konrad Hungerbühler

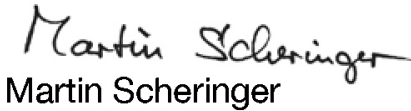
Martin Scheringer

Institute for Chemical and Bioengineering, ETH Zürich, $\mathrm{CH}-8093$ Zürich

The Editorial Board of CHIMIA expresses its thanks to the coordinating guest editors for planning and organising the contributions for the present issue on 'REACH'. 